\title{
MACRO AND MICROSCOPIC MORPHOLOGY OF LYSSA BODY IN DOG
}

\author{
N. Sultana*, M. Afrin, T. Amin and M. Afrose \\ Department of Anatomy and Histology, Faculty of Veterinary Science, Bangladesh Agricultural University, Mymensingh- \\ 2202, Bangladesh
}

\begin{abstract}
With the aim to study the gross and histomorphology of the lyssa body of dog, seven heads were collected from the healthy adult dogs used for dissection purpose in the laboratory of the Department of Anatomy and Histologyat Bangladesh Agricultural University, Mymensingh. The gross parameters such as weight, length and width were recorded for respective specimens. The present study revealed that the dog lyssa was pinkish white coloured and situated along the median plane on the ventral surface of the free portion of the tongue. The lyssa had more or less rod shaped. The weight, length and width of the lyssa were measured as $0.11 \pm 0.01 \mathrm{gm}, 3.96 \pm 0.4 \mathrm{~cm}$ and $0.28 \pm 0.03 \mathrm{~cm}$, respectively. Then the specimens were processed for histomorphology and stained with Hematoxylin and Eosin. The present study noticed that the entire body of the lyssa was formed from adipose tissue and striated muscle bundles, encircled by a thick connective tissue capsule of mainly collagen fibers. These results will be helpful to study the further functional importance of the lyssa body in dog.
\end{abstract}

Keywords: Morphology, lyssa body, dog.

\section{INTRODUCTION}

Lyssa body is a cordlike structure composed offibrous tissue, skeletal muscle and adipose tissue, surrounded by dense irregular connective tissue sheath. It is known to found in carnivores, porcine, camels and pangolin. Embryologically, the lyssa is derived from the neuroectoderm and morphologically, it is situatedin the ventralpartat the apex of the tongue in carnivores, pangolin (Budras et al., 1994; Capellari et al., 2001; Besoluk et al., 2006; Kassem et al., 1984; Prapong et al., 2009). In the ancient times, the lyssa body was considered to be a wormcausing rabiesand the removal of itwas considered to prevent the disease (Easley, 1999).It is about 4 to $5 \mathrm{~cm}$ long in larger dogs (Getty, $5^{\text {th }}$ edition). Some studies on the morphology of lyssa body (Besoluk et al., 2006; Shoeib et al., 2014) havebeen reported in other countries. But no comprehensive study has yet been undertaken on the lyssa body of dog in Bangladesh.

In a comparative morphological studies, Shoeib et al. (2014) claim that the length of the lyssa ranges from 3 to $4 \mathrm{~cm}$ in mixed breed or mongrel dog. An Egyptian scientist's group described the shape of the lyssa was rod whereas a scientist group from Turkey claimed the shape is J-shape.The histological features of the lyssa body presented by Shoeib et al. (2014) and Besoluk et al. (2006) were also conflicting. Since previously described anatomical and histological features of the lyssa body in dog are inconsistent. Therefore, in the present study the authors aimed to describe a detail morphological feature of the lyssa in the dog.

\section{MATERIALS AND METHODS}

The present study was carried out in the laboratory of the Department of Anatomy and Histology, Faculty of Veterinary Science, Bangladesh Agricultural University, Mymensingh-2202, Bangladesh.

\section{Collection of specimens}

The study was performed on seven healthy adult local street dogs of both sexes. The animals were weighing 24 to $28 \mathrm{~kg}$. All the heads were obtained from the recorded corpses used for the practical demonstration ofundergraduate and post graduatestudents in an anatomy laboratory. The collected dogs were anaesthetizedintramuscularly with $2 \mathrm{mg} / \mathrm{kg}$ xylazine $\mathrm{HCl}$ (Rompun ${ }^{\circledR}$, Bayer, Istanbul, Turkey) and $5.5 \mathrm{mg} / \mathrm{kg}$ ketamine $\mathrm{HCl}$ (G-Ketamine ${ }^{\circledR}$, Gonoshasthaya pharmaceuticals Ltd, Bangladesh), and subsequently killed by exsanguination from the right common carotid artery without regaining consciousness. After the tongues were removed from the dogs, the lyssa bodies were resected from the ventral surface of the collected tongues. The collected lyssa bodies were then investigated macroscopically.

\section{Measurement of weight, length and width}

After collecting individually, the lyssa bodies were weighed with the help of an electric balance. The shape of the lyssa body was examined by visual observation. The length and width were measured with the help of measuring scale.

*Corresponding e-mail address: nsultana.bau@gmail.com 


\section{N. Sultana and others}

\section{Histochemistry}

The lyssa body tissues obtained from the dog's tongue were fixed in the formalinsolution for 72 hours and were dehydrated in the series of ascending graded of alcohol followed by clearing in 3 changes in xylene, and the tissues then infiltrated with different graded of melted paraffin in the oven. The tissues were then embedded in paraffin and finally the sections were cut at 5-6 $\mu \mathrm{m}$ (micrometer) thickness using sliding microtome (MIC 509, Euromex, Japan). After cutting, the sections were floated on luke-warm water in a floatation bath at $37^{\circ} \mathrm{C}$ for stretching and then the sections were mounted on clean slides using an adhesive i.e. egg albumin and dried on a slide warmer at $37^{\circ} \mathrm{C}$. The sections were stained using Mayer's Hematoxylin and Eosin (H \& E) for histomorphometrical analyses. The histological structures of the lyssa bodies were observed using light microscope under at $4 \mathrm{X}$ and $10 \mathrm{X}$ magnification. Photographs from the selected specimens were prepared for better illustration of the results.

\section{Data analysis}

All the data's were recorded and presented as mean \pm standard deviation.

\section{RESULTS AND DISCUSSION}

\section{Gross study of the lyssa body}

The lyssa bodies were pinkish coloured structures situated along the median plane on the ventral surface of the apex of the tongue (Figure 1A and B). This finding was similar to the finding of Besoluk et al. (2006) and Shoeib et al. (2014). The shape of each lyssa was just about rod-shaped (Figure 1C and D). This report was supported by the report of Shoeib et al., 2014 and differed from the report of Besoluk et al. (2006). The pan'golin lyssa was also rod shaped structure (Prapong et al., 2009). The caudal one third of the lyssa was located deeply in the midst of the lingual muscle whereas the rostral two third was positioned superficially just under the mucous membrane of the tongue. The weight, length and width of the lyssa were $0.11 \pm 0.01 \mathrm{gm}, 3.96 \pm 0.4 \mathrm{~cm}$ and $0.28 \pm 0.03 \mathrm{~cm}$, respectively. The length of the lyssa was reported $3.5 \mathrm{~cm}$ in adult Turkish dog (Besoluk et al., 2006) and 3-4cm in adult healthy Mongrel dogs (Shoeib et al., 2014). According to the author's knowledge, the weight of the lyssa was not reported yet.
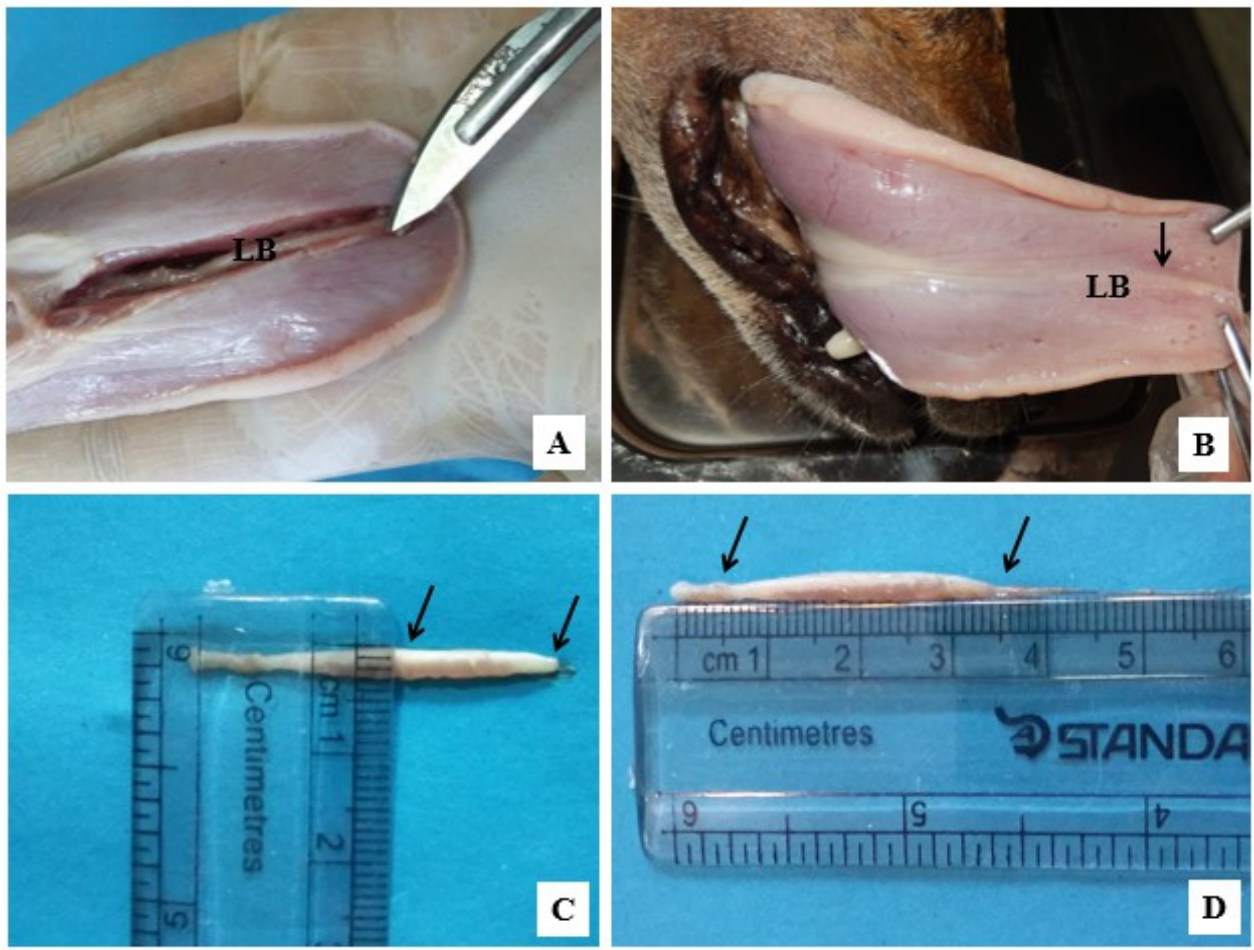

Figure 1. Location of the lyssa body in dog's tongue, arrow shows the lyssa body under the mucous membrane of the tongue (A \& B). Measurement of width and length of lyssa body, arrows show the rod-shaped of lyssa body (C \& D). Abbreviation: LB, Lyssa body. 


\section{Histological study of the lyssa body \\ Sheath of the lyssa}

The transverse section of the lyssa body showed that it was encircled by thick capsule of dense connective tissue (Figure $2 \mathrm{~A}$ and B).The capsule was mainly composed of bundles of collagen fibers. These reports are in agreement with Besoluk et al. (2006) and Shoeib et al. (2014).
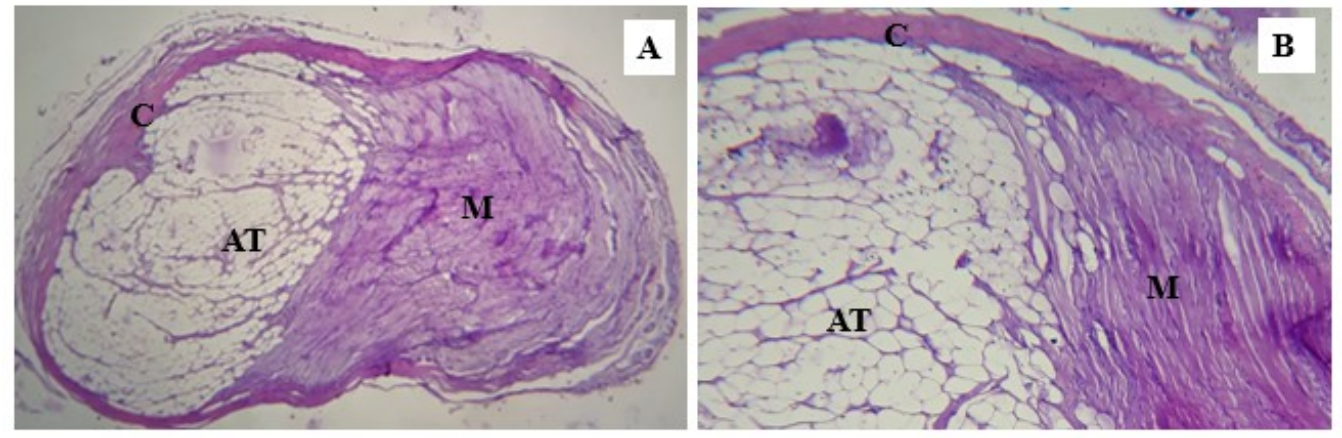

Figure 2. Microscopic representation of lyssa body in dog (A \& B). Abbreviations: C, Connective tissue capsule; AT, adipose tissue; M, skeletal muscle.

\section{Body of the lyssa}

The entire part of the lyssa body was formed from adipose tissue and striated muscle bundles (Figure 2). In the full body of the lyssa, the adipose tissue is situated ventral to the striated muscle bundles and enclosed by the capsule.Moreover, in the adipose tissue mass, scattered muscle fibers, blood vessels and nerve fibers were seen. On the contrary, dispersed adipocytes were found in the striated muscle bundles especially towards the capsule (Figure 2A). This result is similar to the result of Shoeib et al. (2014) but differ from the result of Besoluk et al. (2006). Besoluk et al. (2006) reported that the entire body of the lyssa was composed of more or less adipose tissue with scattered striated muscle, blood vessels and nerve fibers. They also reported that the bundles of striated muscle fibers were situated dorsal to the adipose tissue only in the middle part of the lyssa body.

In the gross and histological studies, the authors of the present research suggested that the lyssa body of the dog serves as an elastic extremity and/or supportive structure of the tip of the tongue. The gross and histological similarities and dissimilarities might be due to the genetic effects of the breed.In the present study, male and female dogs were not considered separately for studying the morphological variations of the lyssa body. The authors assert that further embryological development studies are required to understand thoroughly the functional importance of the lyssa body in dog. The authors also suggest that the results from this study will be constructive for surgical management's i.e. oral tumors, mandibuloectomy, maxillectomy etc. in dog.

\section{REFERENCES}

1. Besoluk K, Eken E and Sur E (2006). Morphological studies on lyssa in cats and dogs. Veterinarni Medicina 51: 485-489.

2. Budras KD, Fricke W and McCarthy PH (1994). Anatomy of the Dog. An Illustrated Text. 3rd ed. Mosby Wolfe, London. 43:109.

3. Capellari H, Egerbacher M, Helmreich and Bock P (2001). Bau und Gewebekomponenten der Lyssa: Fettzellen, myxoideZellen und KnorpelzellenbindenAntikorpergegen S-100 Proteine. Wiener TierarztlicheMonatsschriften 88: $197-202$.

4. Easley K (1999). Veterinary Dentistry: Its origin and recent history. Journal of Dentistry 47: 83-85.

5. Kassem AM, Shahien YM, Kandil SA and Moustafa IA (1984). Histological and histochemical studies on the lyssa of the camels tongue (Camelusdromedarius) during ontogenetic development. Zagazig Veterinary Journal 9: 92-103.

6. Prapong T, Liumsiricharoen M, Chungsamarnyart N, Chantakru S, Yatbantoong N, Sujit K, Patumrattanathan P, Pongket P, Duang-ngen A and Suprasert A (2009). Macroscopic and Microscopic Anatomy of Pangolinûs Tongue. Kasetsart Veterinarians 19: 9-19.

7. Shoeib MB, Rizk AZ and Hassanin AM (2014). Comparative morphological studies on lyssa in carnivores and camels with special reference to its surgical resection. Journal of Advanced Veterinary Research 4: 135-141. 